\title{
Teaching Mathematics Remotely: Changed Practices in Distance Education
}

\begin{abstract}
This investigation explored the challenges of creating meaningful mathematics practices for a community engaged in Distance Education (DE). Specifically, the study maps the influence of new technologies on the practices of a learning community where mathematics was taught remotely. The theoretical framework of this study utilised Bourdieu's work on practice to consider the changed nature of the field, in this case, remote education provision, over time. By using Bourdieu's notion of field, we are better able to understand the ways in which practices and discourses shape particular ways of working in rural education provision. The results of the study show that Field One was innovative and beyond the non-school world, while Field Two lagged behind the technological resources of the non-school world.
\end{abstract}

Over the period of a decade, the advances in digital technologies have created new learning opportunities for distance education provision for rural and remote learners. However, within a Bourdieuian framing, we propose that other practices structure the field which either hinder or enhance learning opportunities. The paper presents two cases of the field in operation. In the first case, the field of distance education employed innovative satellite technologies that were being introduced. We argue that the learning community created by these structuring practices created a range of teaching practices around curriculum delivery, pedagogical approaches, assessment methods and how the community collectively worked to support students' mathematics understandings. The second case, which is of the same site eight years after the introduction of the satellite technology, described how the community's practices were adapted and changed to meet the needs of the students and the wider community in a changed field. The changes in technology create new opportunities through the structuring practices of the field. The paper compares these two fields that have been shaped by the emerging technologies and their impact on learning possibilities for both teachers and students in distance education.

With the changing structuring practices in the distance education field, the paper draws on the ways in which these changes shape and reshape the habitus of the teachers and other stakeholders. In the period of the study, not only were there considerable changes in the digital technologies upon which the practices of the field were founded, but also significant changes that have occurred in the governance of these sites permeated the operations of the sites so as to reconfigure the practices in (potentially) different ways. These changes also impacted the ways in which distance education was offered and the learning potential for students and other stakeholders.

\section{Learning in Remote Communities}

Along with Indigenous students, those students living in rural and remote areas of Australia are among the country's most disadvantaged in terms of most equity measures (Arnold, 2001; Considine \& Zappalà, 2002; Human Rights and Equal Opportunities Commission, 2000). From an educational perspective, rural students' performance on standardised numeracy tests are $28 \%$ below the national average in Grade 3 and this increases to $36 \%$ by Grade 5. For students living in remote areas, the statistics are even more dramatic and concerning (48\% below the national average in Grade 3 and $72 \%$ by Grade 5, MCEETYA, 2005). For students in remote areas, schooling is considerably different - both in context and structure - to the schooling received by the majority of the 
population who live in metropolitan areas. Moreover, the pedagogy required to construct meaningful and engaging learning experiences for these students is, indeed, problematic and challenging.

The educational disadvantage experienced by students living in rural and remote locations has increased despite the attention given to redressing the situation (Lyons, Cooksey, Panizzon, Parnell \& Pegg, 2006; Ryan, 2001). The isolation experienced by these students and their teachers creates challenges that are seldom encountered elsewhere (Du Plessis \& Bailey, 2000). Vast distances between homes and schools, challenging technology and communication issues, minimal face-to-face contact between students and their teacher, and untold social hardship from drought have resulted in situations where more "traditional" teaching and learning is contested (Lowrie, 2007). Moreover, the role of the parent (who is also required to act as an educational supervisor) dictates that mathematics education programs are very different to those "typical" practices which occur in most classrooms (Lowrie, 2006). Even in "best practice" situations, formal partnerships arrangements between teachers, students and their parents rarely move beyond the influence of the classroom teacher (Askew, 2004)-irrespective of how much involvement parents have in the construction of the mathematics curriculum and learning outcomes. In distance settings, the roles of teacher, students and parent are framed within a field that exists within a relatively narrow communication boundary. These limited experiences are intensified in DE settings since the social and cultural dynamics of the classroom are somewhat restricted. Students rarely (if ever) engage or collaborate with one another in terms of mathematics sense making and the opportunity to engage with others from different cultural and socioeconomic backgrounds are relatively restricted. Most social interactions occur when students attend "camps" or mini schools - and generally those limited opportunities are framed around subjects such as physical education, art and drama. Furthermore, the classroom teachers, especially in terms of mathematics, have limited opportunities to provide rich social-cultural experiences because most of their teaching is on a one-to-one basis. These restricted dynamics, if nothing else, create learning contexts different from traditional classrooms where up to 30 different viewpoints are likely to be considered in any mathematics lessons.

For students studying in distance education settings, mathematics is not typically (re)presented is dynamic, socially connected, ways. More visually and communicatively rich technologies were considered to be one way of exposing students to some of the richness available in traditional classrooms (Lowrie, 2006). The body of research which highlights the complex social function of mathematics learning suggests that social-cultural dimensions (Lerman, 2001), gesture (Radford, 2009), collaboration (Goos, 2004), and cultural capacity (Zevenbergen, 2000) are critical in enhancing learning opportunities and sense making.

This investigation describes the changed practice of a distance education learning community over time. Specifically, it considers the influence of technologies within mathematics learning contexts through site-based case studies of a DE school. The study explores the way in which teachers, families and their children create numeracy meaning from contexts that are detached from "regular" classroom experiences. These case studies are framed within Bourdieu's $(1991,1993,1998)$ notion of the field and describe practices which either hinder or enhance learning opportunities. This investigation outlines:

1. the mathematics practices associated with distance education across two fields; and

2. the influence of technological innovations of these practices. 


\section{Theoretical Framework}

In theorising this paper, we draw on the work of French sociologist, Pierre Bourdieu, whose theoretical project allows us to the consider the changing dynamics of the field which is, in this case, the field of distance education, and how these changed fields create different potentialities for the development of capital. In this study, we compare the site of distance education over a period of time. The field of distance education has altered considerably over this timeframe, and we argue that these changes have created different opportunities for learning. Using Bourdieu's theoretical constructs of field, capital and habitus, we propose that there is a dialetic between constructs that must be understood if we are to understand the ways in which learning environments create opportunities for learning, which, in turn, become forms of capital that can be exchanged for objects of value within that field.

According to Bourdieu's tripartite model, the field regulates and is regulated by the practices within that field. Through these practices, participants have greater or lesser potential to accumulate forms of capital that can be exchanged for other rewards within that field. The potential for accumulation of capital is shaped by the habitus of the participant but also the habitus can be shaped by the practices within that field. Bourdieu and Wacquant explain this interplay thus:

People are at once founded and legitimized to enter the field by their possessing a definite configuration of properties. One of the goals of research is to identify these active properties, these efficient characteristics, that is, these forms of specific capital. There is thus a sort of hermeneutic circle: in order to construct the field, one must identify the forms of specific capital that operate within it, and to construct the forms of specific capital one must know the specific logic of the field. (Bourdieu \& Wacquant, 1992, pp 107-108, italics in original)

The field is a site where there are competitive struggles among agents who seek to gain positions of power while positioning others. Through these struggles, the habitus of the agents can be revealed. In education the mythology that the classroom context is a site for apolitical knowledge production assumes an almost unchallenged status. A liberal view is that education is a site for creating knowledge among learners who may be more or less successful in that knowledge creation. But within Bourdieu's theoretical project, the classroom becomes a site where there is a clear political process where agents and structures interact. Through this interaction, some students are more or less likely to be successful. Schooling is not an apolitical process, but through the structures within the field, some students will enter the field with greater or less capital as part of their habitus. Through the practices within a given field, these dispositions are given greater or lesser value and hence the different trajectories of learners are shaped by the practices of a field. Bourdieu is not structuralist or deterministic in his approach but sees that there is considerable scope for change.

Education is an important field because of its capacity to confer capital, particularly cultural capital, upon its participants. Indeed, education can be referred to as an academic market in terms of its distribution of such cultural capital. (Webb, Schirato \& Danaher, 2002, p. 110)

What becomes important according to Bourdieu's work is that as students enter the field of education, their habitus may align more or less with the practices of the field. Those dispositions within the habitus that align with the field may be seen as forms of capital - in this case, cultural capital, where cultural dispositions have been embodied by the learner and so position him/her more favourably within that field. Being able to speak the language of the classroom, having knowledge that is valued within that field, and 
embodying cultural dispositions that are strongly aligned with the field make for greater opportunities for being positioned as a successful learner. The nature and function of this field is distinctive in distance education settings. The task for educators is for the reconstitution of the habitus of some learners so that it more strongly aligns with the practices within that field. When this occurs, the teacher is able to confer status on the learner through a range of structuring practices that reify the culture as a form of academic success.

This differential recognition of habitus is described by Bourdieu and Wacquant as follows:

...social agents are not "particles" that are mechanically pushed and pulled about by external forces. They are, rather, bearers of capital and, depending of their trajectory and on the position they occupy in the field by virtue of their endowment (volume and structure) in capital, they have a propensity to orient themselves actively either toward the preservation of the distribution of capital or toward the subversion of this distribution. (Bourdieu \& Wacquant, 1992, pp. 108-109, italics in original)

Mills (2008) draws attention to what she refers to as reproductive and transformative habitus. In her work with rural students, Mills argued that where students resign themselves to the systemic failure of education, the reproductive habitus enables the reproduction of social disadvantage. However, the transformative habitus is one where the learners are proactive in reconstituting their habitus and thus enabling greater educational opportunity and outcomes. The art of good education is the potential of teachers to bring about a transformative habitus so that learners can feel empowered to engage with quality learning that will enable them to transition successfully from their marginalised social position to one of greater access and success in schooling and beyond. However, to assume that the teacher is solely responsible for such transformative practice belies the complexity of the learning context, so it is critical to understand the intersection of these variables. The teacher can be constrained by the structuring practices within the field and these contribute to the potentiality of transformative education.

We draw extensively on this theoretical position to better understand the changing practices within distance education, and how such changes impact on the learning, and learning outcomes of rural/remote students who, by nature of their geographical location, participate in distance education learning environments. To apply Bourdieu's constructs to the field of distance education, we are better able to understand how the changing practices within the field are creating environments that may work against the success of young learners and teachers.

\section{Method}

This investigation provides an in-depth case study of a learning community where children learn through distance education. The first phase outlines the field of distance education at a time when innovative satellite technologies were being introduced. It considers and describes the role of the particular distance education site that delivers instruction to these families in order to provide a comprehensive context for the study. Initially, interviews with eight key personnel from the distance education providers were conducted to gather information on the range and nature of services offered for the support of mathematics teaching and learning. These personnel included the executive director of distance education, the principal of distance education school and classroom teachers. Interviews were also conducted (at the residential school) with 12 parents with longstanding associations with this school. The four home supervisors were interviewed 
and observed in the home on a typical school day. Moreover, time was spent watching the students engaged in a range of numeracy practices as the supervisor made sense of the curriculum. Data from each home site were combined with data from the interviews with personnel from the schools of distance education to form a rich contextualisation of the field. The first aspect of the field described effective strategies, processes and pedagogies in numeracy development of these rural and remote students. A Home Supervisor Interview Schedule was developed to initiate a conversation with the home supervisor in order to ascertain the influence the program had on both the pedagogical practice of the program and the relationship initiatives that emerged from the program. In addition, a School Interview Schedule was developed to ascertain teachers' views of the program and to gain insights into how they developed learning partnerships with families.

The second aspect of the field described the case study site eight years after the initial data collection phase. The principal of the school was re-interviewed in order to ascertain the changed practices within the field concerning policies, teacher pedagogies and students participating in the distance education environment. A focus group with ten primary and secondary mathematics teachers was conducted after the development of a semi-structured interview schedule. The focus group considered issues associated with the nature of teaching and learning environments, professional development experiences, technology challenges, student-teacher interactions, and teachers' current pedagogical practices. A follow up site visit was also conducted where additional questions which arose from the focus group were both clarified and expanded upon. In addition, mathematics lessons were simultaneously observed from both the school site and a remote home site (hundreds of kilometres from the school). Detailed observation schedules were completed during these site visits.

\section{Results}

\section{Field 1: Distance learning with new technological innovations}

Distance education, in the state of New South Wales, Australia, is coordinated from a centralised unit which was responsible for eight distance education schools. The distance education learning community which formed this case study site is structured quite differently to more typical school contexts. The face-to-face learning environment which surrounds most classroom practices is not present in distance education learning environments. Although the actual components which make up these learning environments are similar-including policies, resources, curriculum content and indeed all other aspects of a learning community - the impact and connectivity of these components are both distinct and variously influential. For example, if available technologies do not work well in the traditional classroom setting, other plans can be made, whereas in a distance education setting, the lesson cannot proceed. Furthermore, interactions between students in the "classroom" bear little resemblance to interactions within a conventional classroom, and therefore the process of engagement has to be thought about differently.

The classroom teacher generally has a smaller number of children in their class (typically 14 in primary school). These children are usually hundreds of kilometres away from the school and may only see the teacher (in a physical sense) three or four times per year. Children in the class may see one another less than this. The advent of new technologies created opportunities for children and their teachers to engage and interact with one another beyond the static and one-way opportunities previously available. With radio communication systems for example, only one person could talk at a time, with 
protocol in place to ensure that no one ever interjected in ways that could commonly occur in traditional classroom contexts. With email and video capacity, students could simultaneously respond to one another and to the questions the classroom teacher posed. Although this new form of communication could not be considered dynamic, it did provide different opportunities for learning to occur. These new classroom interactions were scheduled only twice a week and therefore a transmissive model of one-way communication was still dominant. Nevertheless, there seemed to be great hope for new forms of learning to take place since teleconferencing, audio-graphic conferencing, and satellite lessons were now available.

One of the main differences between regular and distance education schools involves the implementation of learning activities and, in some cases, the development of learning programs by each child's home supervisor. In most cases, the supervisor responsible for supporting student/teacher interaction was the mother of the child who must interpret the planned program and guide its implementation. In order to assist supervisors with program implementation, distance education teachers prepare accompanying notes for home supervisors on the planned program. Teachers encourage home supervisors and students to make regular contact with them during the school day-usually via telephone or email. A vital partnership between the teacher and supervisor was established through such engagement. This is not to say that parents in traditional classroom contexts do not have a strong influence over their child's education, but this explicit and daily communication was undertaken as part of the formal learning process. What we found as noteworthy was that the supervisor still had the duel function of being a parent.

Most curriculum content was developed and assigned to the students over a two-week period and included workbooks, the home supervisor guide, and accompanying resources. Ideally, these resources arrived at least one week before the current unit had been completed in order to provide home supervisors with sufficient time to prepare for unit implementation. Units of work, usually presented in themes, were common and obviously help with resourcing. For example, a theme on farm animals spread across literacy, social science and science curriculum areas. By contrast, mathematics units were not theme based and nor were they strand based. Consequently, the students may have been undertaking number-based activities on Monday through to Wednesday with measurement and spatial concepts addressed on Thursday and Friday respectively. This learning sequence was typical of many traditional classrooms at this time. Supervisors would receive a substantial mathematics resource kit that was used throughout the year. This kit included MultiAttribute Blocks (MAB), counters, measurement devices, number line cards and an extensive array of mathematics puzzles and games. In addition, specific resources (e.g., counting frames for lessons on addition or Tangrams for spatial transformation activities) aligned to the fortnightly "themes" were received and then sent back to the school with the work completed during two weeks. Home supervisors were expected to correct student work when completed and write accompanying comments both for the student and teacher. Completed student workbooks were often returned with accompanying artefacts including photos, cassette recordings, and drawings, as a means of further evidence of student learning. These records of student activity were of great benefit to the classroom teacher since this was their primary source of information to assess student progress.

\section{The introduction of satellite communications}

The introduction of satellite technology was viewed, by most parents and teachers, as an opportunity to completely reshape the distance education learning environment. For 
parents, in both their role as parents and supervisor, these new technologies were regarded as a support system which better replicated what would happen in a traditional classroom context - especially when children were being introduced to new mathematics concepts that they themselves were not comfortable in explaining. Furthermore, they thought it particularly valuable to have visual cues from teachers when demonstrating how to use mathematics materials (e.g., MABs) in order to support students' conceptual development. Previously, the supervisor relied on instructions given in printed materials (that came with the resource tubs) to appreciate the philosophy and instructional intent of such resources. Interestingly, the supervisors felt that the increased communication capacity of the technology would be more beneficial to them in their supervisory role than it would for teachers in their role of engaging with students.. Since most supervisors (more than 85\%) did not have any formal teaching experience or qualifications the prospect of being able to observe (visually with the new technology) how the classroom teacher was actually delivering lessons was regarding as being invaluable. The opportunity to observe the student/teacher interaction in a more dynamic (multimodal) form was considered to be a form of professional development for the supervisor. At this point in time, supervisors did not have access to any formal professional development.

Most supervisors felt that the increased opportunities that would result in the new technologies would broaden the richness of classroom interactions and engagement. By contrast, they did not assume, or consider it necessary, that increased capacity for internet use would be a beneficial supplement to satellite capabilities. This was based on the belief that the resources supplied by the school, the teaching materials they could access and current teacher delivery were sufficient to students needs. Although the teachers recognised the steep learning curve they faced in utilising effectively these new technologies, they too had a high disposition for engaging with this medium of lesson delivery. Teachers were of the view that an increased technology capacity would allow learning material to be modified to students' needs more easily or at least remain more current in their presentation. Some of the materials being used in the school were written more than 25 years ago.

What can be seen in this context is how the structuring practices of the field, such as the emerging satellite technologies or the standard mathematics equipment made available to parents, as well as a curriculum of a substantial age, offered particular learning experiences to the various stakeholders, including students, supervisors and teachers. These structuring practices created various potentialities for restructuring the habitus. Parents, for example, were exposed to the new technologies that enabled them to better understand the ways in which mathematics learning could be facilitated. Thus, the embodiment of mathematics practices and culture into a mathematical habitus was being made possible through these structuring practices for rural/distance learners.

\section{Field 2: Distance learning beyond technological innovations}

The governance of distance education had changed since undertaking the initial investigation in Field 1. Within this state jurisdiction, the organisation of distance education had moved from a centralised- to a regional-governance approach. As a consequence, the technology capability of the school and its ongoing influence on mathematics had not been as influential as first anticipated in the eight years since the technology was rolled out. Increasingly, the school was responsible for the development of mathematics teaching programs rather than a collective approach as described in Field 1 where the program was formulated elsewhere. By way of example, the school was actually 
now asked to pay the costs of printed materials from their own budget rather than such learning materials being supplied by a centralised body.

Initially the school community maintained that new technologies would reshape practice. Such hopes were probably a reflection of what society in general thought new technologies would bring to the future. Although both teachers and parents thought that children would be eager to use the technology regularly, this has not been the case. Children are often more willing to complete tasks in the pencil-and-paper form since they prefer the "concreteness and physicality" of this medium. Consequently, a more blended form of learning has been established.

Teachers have changed their practice to accommodate the technology rather than let the technology lead curriculum development. This approach has been established for a number of reasons. First, an overall reliance on technology was viewed to be problematic given the unreliability of access in terms of both functionality and bandwidth capacity. Second, many of the technology-based resources promoted a drill and practice mentality where students were more inclined to guess answers rather than think through the mathematical processes. Given the nature of delivery, teachers felt it important to have students represent their work in a pencil-and-paper form so teachers could have some insights into how the students worked out solutions. In other words, teachers' practices required concrete representations that could help them assess students' learning and at present, this type of facility is not available in technology-based delivery. Third, there is a view that even reliable technology resources (e.g., Moodle) needed to be presented in a blended form. For example, information which is presented in an online form was more valuable when printed materials were part of the learning experience. Finally, most assessment practices around Australia rely on pencil-and-paper assessment (e.g., National Assessment Programme - Literacy and Numeracy [NAPLAN]) and therefore teachers felt that students had to become familiar with such types of representations.

Although the two-way radio technology is antiquated, parents particularly missed this form of interaction. Internet reliability meant that it was challenging to email other parents and long telephone conversations were a cost issue. The ease with which parents could communicate had diminished. When the satellite technology was introduced, it was restricted to students in primary schools. Surprisingly, this technology capability is yet to be afforded to secondary students. In terms of the delivery of mathematics, teachers felt it was more appropriate to interact with the children on a one-to-one basis through daily telephone conversations. Although it was acknowledged that the students did not have the opportunity to engage with other students in their mathematics lessons, the opportunity to target students at their current level of development was viewed to be more important and beneficial.

One of the major changes in the delivery of mathematics has been the move from a designated supplier of content materials. In Field 1, learning materials were solely sourced from one provider (Learning Materials Production Unit, Education Queensland). Teachers delivered units from these artefacts and varied lessons only slightly. By contrast, this blended learning framework sources learning materials from a number of suppliers including TaLe (New South Wales Department of Education and Training [NSW DET]), McDonalds Maths online (http://mathsonline.com.au/) and the Rigby Maths Tracks (Leigh, 2008) program. These resources provide both online and printed materials with teachers creating individualised mathematics programs for students. In Field 2, teachers are required to develop their own resources for a variety of media and present those materials in multiple ways including through print, electronically, or on the web. To this point, the 
teacher's role has changed markedly. Although it appears that such flexibility provides students with increased access to resources, and to some extent promotes more open-ended and flexible learning investigations, the actual practice more reflects teachers creatively weaving ideas and learning opportunities into individualised learning programs. Therefore, part of the changed practice involves teachers selecting and matching resources for students (and thus a changed practice). Nevertheless, students still receive "a package," albeit one that is more blended in nature. It is noteworthy that one learning "series" has been replaced with a different mathematics resource - and this new resource is not one that was specifically designed for distance education. Parents, in particular, maintained that this new direction resulted in a less comprehensive package - and as such the students were more reliant on them, and their teachers, to complete assigned work. This shift in the structuring practices of the field manifest in the subjective structuring practices. In this instance, the shift in locus of control from the external resources to the parents meant that parent felt less confident in supporting their children's learning.. By losing this confidence in how to teach due to the change in resources, parent felt disempowered and, hence, not as willing to engage as they had previously been. This subjectivity manifest in their beliefs about their capacity to confidently teach mathematics to their children.

The communication and technology advances of recent years are not the fundamental initiator in the creation of individualised learning programs. Changes in student composition, and the redefined nature of learning contexts, have influenced the move toward specialised programs. More students are now enrolling into DE for reasons other than their rural/remote circumstances. For example, students deemed to be behaviourally challenging, or students with disabilities, account for a high proportion of enrolments. Moreover, rural populations have changed markedly over the past eight years with fewer small farms and fewer people working these farms. There is also a high proportion of Indigenous students in the school. Distance education has become specialised educationwhich is quite appealing for parents who feel their children do not "fit" mainstream schooling. Interestingly, it appears that these parents expect high levels of structure within lessons (and indeed the curriculum). Many of the students are challenging to supervise in the home, and parents appreciate the structure involved in routines. In contexts where technology is unreliable, and is embedded within a paradigm that relies on students being able to multitask and work dynamically, work books and pencil-and-paper-based mathematics activities satisfy their needs and expectations.

\section{Discussion}

The outcomes of this study are drawn together using Bourdieu's theoretical framing as a way of making sense of the altered practices within the field and the impact that these changes have on quality learning opportunities for rural and remote students who undertake distance education. In a policy environment that promotes a range of learning opportunities for students and is cognisant of the needs and performances of rural and remote students, the paper raises serious concerns about the changes of the field of distance education and the subsequent learnings being made possible through these changes.

The re-framing of distance learning due, in part, to the changed enrolment patterns of students, has created significant shift in practices. In Field 1, under different conditions and circumstances, there were high levels of access to new technologies, with teachers, parents and students developing high expectations for how these technologies would positively impact on practices. Indeed, all members of the learning community had high expectations for the changed learning environment, with a strong desire to embrace and utilise the 
technologies to best effect. In Field 2 (see Figure 1), there was a dramatic shift in access to these technologies, and the communities' expectations for the technology and their willingness to operationalise their practices within the field had become far less positive.

\section{Insert Figure 1 about here}

What we observe in these two fields are quite different practices. In the first field, the student population was relatively homogenous. They came to DE with similar habitusthat is, a rural habitus - and as such, the teachers' role was to reconstitute that habitus into one which was more transformative in orientation. This enabled the learners (children and parents) to engage with mathematical ideas so that the home habitus would align more strongly with the school mathematics habitus. The possibilities brought about through the satellite technology was more enabling than the practices of the past, but also brought with it challenges for the teachers. They, too, had to reconstitute their teaching habitus to align with the changing demands of this new digital field. Collectively, these changes to the field and the practices within Field One, we would contend, were more enabling in bringing about mathematical habitus of learners that aligned with the field, thus positioning learners more favourably with the objective structuring practices of the field as represented through various objects including testing schemes.

In contrast, in Field Two, we observe that the population attending DE has changed considerably. It now incorporates not only rural learners, but learners from a range of backgrounds disconnected with rural contexts. This diversity creates very different teaching environments, thus making it difficult for teachers to use the pedagogical tools that they had been able to employ in Field One. Whole-class teaching had shifted to oneon-one teaching which creates very different opportunities for mathematical learning and shaping of mathematical habitus. In this case, learners are being exposed to practices that create spaces for constituting mathematical habitus that have more transmissive and individual orientations than in Field One. What this offers are the more traditional habitus of mathematics. In line with the argument early in this paper, this creates a much more reproductive habitus where learners are being locked into old practices and, hence, old habitus, ones which the field of mathematics education has been seeking to address for a number of decades. The structuring practice of one-on-one teaching is failing to include the socio-cultural learning contexts that are so much a feature of contemporary mathematics education.

The inability for the technological structuring practices of Field Two to have changed in the period of observations, suggests that there has been a hiatus in the field which has failed to move with the rapid changes in the non-school world. Where Field One was innovative and beyond the non-school world, Field Two lags behind the technological resources of the non-school world. As such, the technology became a structuring practice which is not abreast with the external world and thus, again, offered reproductive rather than transformative opportunities for DE students.

What we have sought to bring out in this paper is that the changed circumstances of DE provision create different learning opportunities for students. The changes in the two fields have been shaped by a range of factors, not least of which are the technological innovations and the changing clientele of DE. Collectively these offer different potentialities for learning, and thus habitus creation (or reconstitution). Given that rural and remote students have been identified as groups of learners who are significantly at risk of poor performance in mathematics, we contend that there are structuring practices that are 
offering enhanced and/or restricted potentialities for learning mathematics. We have found that using Bourdieu's theoretical framing has allowed us to theorise this situation and to argue that the changes in the two fields need to be considered carefully for their ramifications for learning and access to school mathematics for students in distance education contexts. 


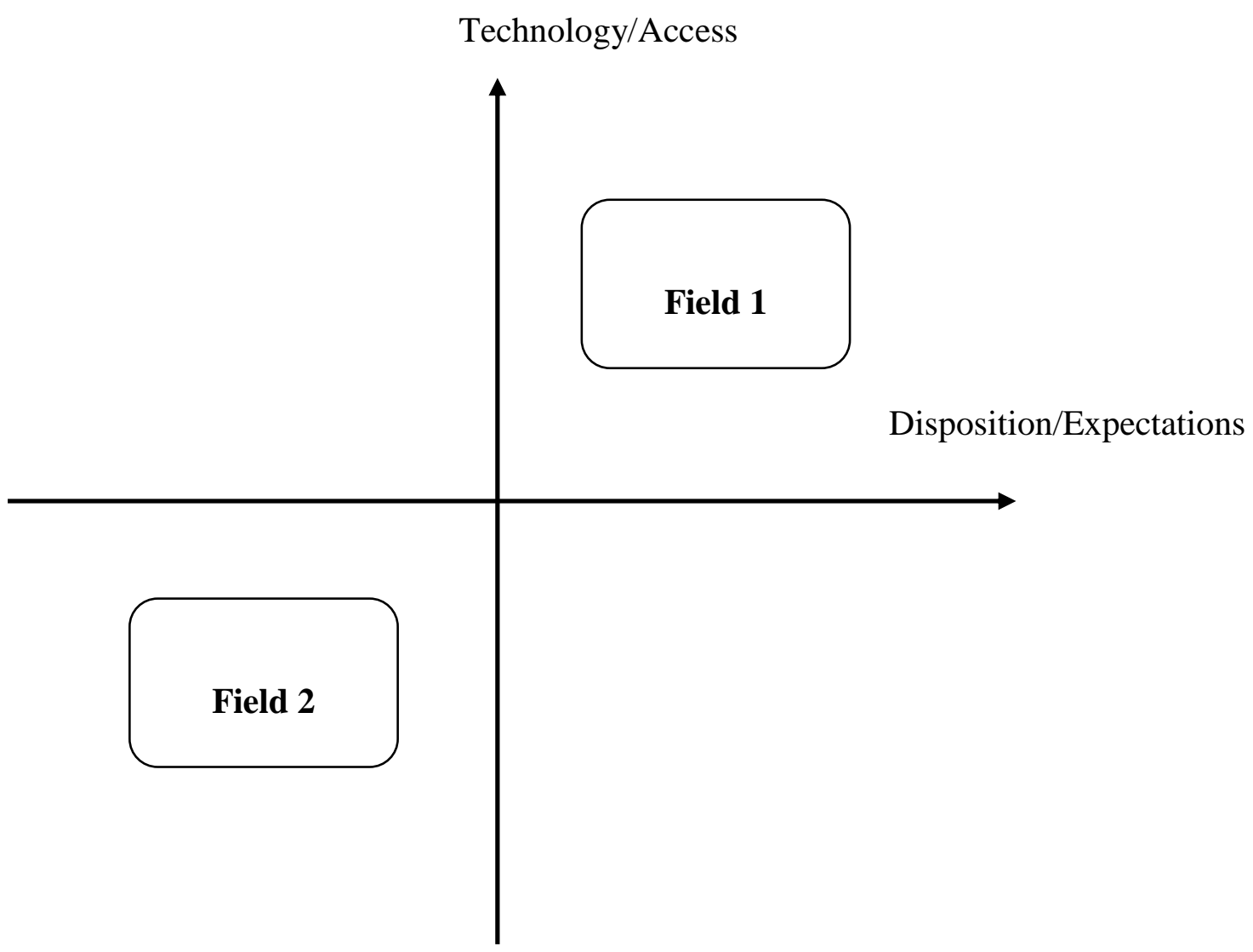

Figure 1. The Repositioning of "Field" within Distance Education practices in Australia. 


\section{References}

Arnold, P. (2001). Review of contemporary issues for rural schools. Education in Rural Australia, 11 (1), 31 42.

Askew, M. (2004). Teaching and learning primary numeracy. Address presented to the Special Topic Group 1 at the $10^{\text {th }}$ International Congress on Mathematical Education, Copenhagen, Denmark.

Bourdieu, P. (1991). Language and symbolic power. Cambridge, MA: Harvard University Press.

Bourdieu, P. (1993). Sociology in question. London: Sage.

Bourdieu, P. (1998). Practical reason. Cambridge: Polity Press.

Bourdieu, P. \& Wacquant, L. J. D. (1992). An invitation to reflexive sociology. Chicago: Chicago University Press

Considine, G., \& Zappalà, G. (2002). The influence of social and economic disadvantage in the academic performance of schools students in Australia. Journal of Sociology, 38 (2), 129-148.

Du Plessis, D., \& Bailey, J. (2000). Isolated parents' perceptions of the education of their children. Education in Rural Australia, 10, 1-26.

Goos, M. (2004). Learning mathematics in a classroom community of inquiry. Journal for Research in Mathematics Education, 35(4), 258-291.

Human Rights and Equal Opportunities Commission [HREOC]. (2000). Emerging themes: National inquiry into rural and remote education. Sydney, Australia: HREOC.

Human Rights and Equal Opportunity Commission [HREOC]. (2000). Recommendations: National inquiry into rural and remote education. Sydney, Australia: HREOC.

Leigh, T. (2008). Maths Tracks NSW series. Melbourne, Australia: Pearson Education.

Lerman, S. (2001). Cultural, discursive psychology: A sociocultural approach to studying the teaching and learning of mathematics. Educational Studies in Mathematics, 46, 87-113.

Lowrie, T. (2007). Learning engagement in distance and rural settings: Four Australian cases. Learning Environments Research, 10, 35-51.

Lowrie, T. (2006). Establishing school-family partnerships in distance education contexts: Pedagogical engagement in isolated settings. Journal of Distance Education, 21(2), 96-114.

Lyons, T., Cooksey, R., Panizzon, D., Parnell, A., \& Pegg, J. (2006). Science, ICT and mathematics education in rural and regional Australia: The SiMERR national survey. Canberra, ACT: Department of Education, Science and Training.

Mills, C. (2008). Opportunity and resignation within marginalised students: towards a theorisation of the reproductive and transformative habitus. Critical Studies in Education, 49(2), 99-111.

Ministerial Council on Education, Employment Training and Youth Affairs (MCEETYA). (2005). National report on schooling in Australia 2005. Carlton South, Vic: Author.

New South Wales Department of Education and Training [NSW DET]. Teaching and Learning Exchange. [TaLe] Accessed 10 June 2010 from http://www.tale.edu.au

Radford, L. (2009). Why do gestures matter? Sensuous cognition and the palpability of mathematical meanings. Educational Studies in Mathematics, 70, 111-126.

Ryan, R. (2001). Human rights, remote Australia, and the VET sector. Australian Training Review, 40, 2829.

Webb, J., Schirato, T., \& Danaher, G. (2002). Understanding Bourdieu. Thousand Oaks, CA: Sage.

Zevenbergen. R. (2000). "Cracking the code" of mathematics classrooms: School success as a function of linguistic, social and cultural background. In J. Boaler (Ed.), Multiple perspectives on mathematics teaching and learning (pp. 201-224). Westport, CT: Ablex publishing. 